\title{
Growth Rates of Meromorphic Functions Focusing Relative Order
}

\author{
Sanjib Kumar Datta, ${ }^{1}$ Tanmay Biswas, ${ }^{2}$ and Sarmila Bhattacharyya ${ }^{3}$ \\ ${ }^{1}$ Department of Mathematics, University of Kalyani, Kalyani, Nadia District, West Bengal 741235, India \\ ${ }^{2}$ Rajbari, Rabindrapalli, R.N. Tagore Road, P.O. Krishnagar, P.S. Kotwali, Nadia District, West Bengal 741101, India \\ ${ }^{3}$ Jhorehat F. C. High School for Girls, P.O. Jhorehat, P.S. Sankrail, Howrah District, West Bengal 711302, India
}

Correspondence should be addressed to Sanjib Kumar Datta; sanjib_kr_datta@yahoo.co.in

Received 17 November 2013; Accepted 15 January 2014; Published 2 March 2014

Academic Editors: Y. Latushkin and R. Mallier

Copyright (c) 2014 Sanjib Kumar Datta et al. This is an open access article distributed under the Creative Commons Attribution License, which permits unrestricted use, distribution, and reproduction in any medium, provided the original work is properly cited.

A detailed study concerning some growth rates of composite entire and meromorphic functions on the basis of their relative orders (relative lower orders) with respect to entire functions has been made in this paper.

\section{Introduction, Definitions and Notations}

Let $f$ be a meromorphic and let $g$ be an entire function defined in the open complex plane $\mathbb{C}$ and $M_{g}(r)=$ $\max \{|g(z)|:|z|=r\}$. If $g$ is nonconstant, then $M_{g}(r)$ is strictly increasing and continuous and its inverse $M_{g}^{-1}(r):(|g(0)|, \infty) \rightarrow(0, \infty)$ exists and is such that $\lim _{s \rightarrow \infty} M_{g}^{-1}(s)=\infty$. We use the standard notations and definitions in the theory of entire and meromorphic functions those are available in $[1,2]$.

To start our paper we just recall the following definitions.

Definition 1. The order $\rho_{f}$ (the lower order $\lambda_{f}$ ) of an entire function $f$ is defined as

$$
\rho_{f}=\limsup _{r \rightarrow \infty} \frac{\log ^{[2]} M_{f}(r)}{\log r}\left(\lambda_{f}=\liminf _{r \rightarrow \infty} \frac{\log ^{[2]} M_{f}(r)}{\log r}\right) .
$$

If $f$ is a meromorphic function, one can easily verify that

$$
\rho_{f}=\limsup _{r \rightarrow \infty} \frac{\log T_{f}(r)}{\log r}\left(\lambda_{f}=\liminf _{r \rightarrow \infty} \frac{\log T_{f}(r)}{\log r}\right),
$$

where $T_{f}(r)$ is the Nevanlinna's characteristic function of $f$ (cf. [1]).
Bernal $[3,4]$ introduced the definition of relative order of an entire function $f$ with respect to another entire function $g$ denoted by $\rho_{g}(f)$ as follows:

$$
\begin{aligned}
\rho_{g}(f) & =\inf \left\{\mu>0: M_{f}(r)<M_{g}\left(r^{\mu}\right) \forall r>r_{0}(\mu)>0\right\} \\
& =\limsup _{r \rightarrow \infty} \frac{\log M_{g}^{-1} M_{f}(r)}{\log r} .
\end{aligned}
$$

The definition coincides with the classical one [5] if $g(z)=$ $\exp z$.

Similarly, one can define the relative lower order of an entire function $f$ with respect to another entire function $g$ denoted by $\lambda_{g}(f)$ as follows:

$$
\lambda_{g}(f)=\liminf _{r \rightarrow \infty} \frac{\log M_{g}^{-1} M_{f}(r)}{\log r} .
$$

Extending this notion, Lahiri and Banerjee [6] introduced the definition of relative order of a meromorphic function with respect to an entire function in the following way. 
Definition 2 (see [6]). Let $f$ be any meromorphic function and let $g$ be any entire function. The relative order of $f$ with respect to $g$ is defined as

$$
\begin{aligned}
\rho_{g}(f) & =\inf \left\{\mu>0: T_{f}(r)<T_{g}\left(r^{\mu}\right) \forall \text { large } r\right\} \\
& =\limsup _{r \rightarrow \infty} \frac{\log T_{g}^{-1} T_{f}(r)}{\log r} .
\end{aligned}
$$

Likewise, one can define the relative lower order of a meromorphic function $f$ with respect to an entire function $g$ denoted by $\lambda_{g}(f)$ as follows:

$$
\lambda_{g}(f)=\lim _{r \rightarrow \infty} \inf _{n} \frac{\log T_{g}^{-1} T_{f}(r)}{\log r}
$$

It is known (cf. [6]) that if $g(z)=\exp z$, then Definition 2 coincides with the classical definition of the order of a meromorphic function $f$.

In this paper we wish to prove some results related to the growth rates of composite entire and meromorphic functions on the basis of relative order (relative lower order) of meromorphic functions with respect to an entire function.

\section{Lemmas}

In this section we present some lemmas which will be needed in the sequel.

Lemma 1 (see [7]). Let $f$ be meromorphic and let $g$ be entire and suppose that $0<\mu<\rho_{g} \leq \infty$. Then for a sequence of values of $r$ tending to infinity,

$$
T_{f \circ g}(r) \geq T_{f}\left(\exp \left(r^{\mu}\right)\right)
$$

Lemma 2 (see [8]). Let $f$ be meromorphic and let $g$ be entire such that $0<\rho_{g}<\infty$ and $0<\lambda_{f}$. Then for a sequence of values of $r$ tending to infinity,

$$
T_{f \circ g}(r)>T_{g}\left(\exp \left(r^{\mu}\right)\right)
$$

where $0<\mu<\rho_{g}$.

Lemma 3 (see [9]). Let $f$ be a meromorphic function and let $g$ be an entire function such that $\lambda_{g}<\mu<\infty$ and $0<\lambda_{f} \leq$ $\rho_{f}<\infty$. Then for a sequence of values of $r$ tending to infinity,

$$
T_{f \circ g}(r)<T_{f}\left(\exp \left(r^{\mu}\right)\right)
$$

Lemma 4 (see [9]). Let $f$ be a meromorphic function of finite order and let $g$ be an entire function such that $0<\lambda_{g}<\mu<\infty$. Then for a sequence of values of $r$ tending to infinity,

$$
T_{f \circ g}(r)<T_{g}\left(\exp \left(r^{\mu}\right)\right)
$$

\section{Theorems}

In this section we present the main results of the paper.

Theorem 1. Let $f$ be a meromorphic function and let $g, h$ be any two entire functions such that

(i) $\liminf _{r \rightarrow \infty} \frac{\log T_{h}^{-1}(r)}{(\log r)^{\alpha}}=A$, a real number $>0$,

(ii) $\liminf _{r \rightarrow \infty} \frac{\log T_{h}^{-1} T_{f}\left(\exp r^{\mu}\right)}{\left(\log T_{h}^{-1}(r)\right)^{\beta+1}}=B, \quad$ a real number $>0$,

for any $\alpha, \beta$, $\mu$ satisfying $0<\alpha<1, \beta>0, \alpha(\beta+1)>1$, and $0<\mu<\rho_{g} \leq \infty$. Then

$$
\rho_{h}(f \circ g)=\infty .
$$

Proof. From (i) we have for all sufficiently large values of $r$ that

$$
\log T_{h}^{-1}(r) \geq(A-\varepsilon)(\log r)^{\alpha}
$$

and from (ii) we obtain for all sufficiently large values of $r$ that

$$
\log T_{h}^{-1} T_{f}\left(\exp r^{\mu}\right) \geq(B-\varepsilon)\left(\log T_{h}^{-1}(r)\right)^{\beta+1} .
$$

Also $T_{h}^{-1}(r)$ is an increasing function of $r$ it follows from (13) and (14) and Lemma 1 for a sequence of values of $r$ tending to infinity that

$$
\log T_{h}^{-1} T_{f \circ g}(r) \geq \log T_{h}^{-1} T_{f}\left(\exp \left(r^{\mu}\right)\right)
$$

that is,

$$
\log T_{h}^{-1} T_{f \circ g}(r) \geq(B-\varepsilon)\left(\log T_{h}^{-1}(r)\right)^{\beta+1}
$$

that is,

$$
\log T_{h}^{-1} T_{f \circ g}(r) \geq(B-\varepsilon)\left[(A-\varepsilon)(\log r)^{\alpha}\right]^{\beta+1}
$$

that is,

$$
\log T_{h}^{-1} T_{f \circ g}(r) \geq(B-\varepsilon)(A-\varepsilon)^{\beta+1}(\log r)^{\alpha(\beta+1)}
$$

that is,

$$
\frac{\log T_{h}^{-1} T_{f \circ g}(r)}{\log r} \geq \frac{(B-\varepsilon)(A-\varepsilon)^{\beta+1}(\log r)^{\alpha(\beta+1)}}{\log r}
$$

that is,

$$
\begin{aligned}
\limsup _{r \rightarrow \infty} & \frac{\log T_{h}^{-1} T_{f \circ g}(r)}{\log r} \\
& \geq \lim _{r \rightarrow \infty} \inf \frac{(B-\varepsilon)(A-\varepsilon)^{\beta+1}(\log r)^{\alpha(\beta+1)}}{\log r} .
\end{aligned}
$$

Since $\varepsilon(>0)$ is arbitrary and $\alpha(\beta+1)>1$, it follows from above that

$$
\rho_{h}(f \circ g)=\infty .
$$

This proves the theorem. 
In the line of Theorem 1 and with the help of Lemma 2 one may state the following theorem without its proof.

Theorem 2. Let $f$ be a meromorphic function and let $g, h$ be any two entire functions such that $0<\rho_{g}<\infty$ and $0<\lambda_{f}$. Further suppose that

(i) $\liminf _{r \rightarrow \infty} \frac{\log T_{h}^{-1}(r)}{(\log r)^{\alpha}}=A$, a real number $>0$,

(ii) $\liminf _{r \rightarrow \infty} \frac{\log T_{h}^{-1} T_{g}\left(\exp r^{\mu}\right)}{\left(\log T_{h}^{-1}(r)\right)^{\beta+1}}=B$, a real number $>0$

for any $\alpha, \beta, \mu$ satisfying $0<\alpha<1, \beta>0, \alpha(\beta+1)>1$ and $0<\mu<\rho_{g}$. Then

$$
\rho_{h}(f \circ g)=\infty .
$$

Theorem 3. Let $f$ be a meromorphic function and let $g, h$ be any two entire functions such that

(i) $\lim _{r \rightarrow \infty} \inf _{\log T_{h}^{-1}\left(\exp \left(r^{\mu}\right)\right)}=A$, a real number $>0$,

(ii) $\liminf _{r \rightarrow \infty} \frac{\log \left[\log T_{h}^{-1}\left(T_{f}\left(\exp r^{\mu}\right)\right) / \log T_{h}^{-1}\left(\exp r^{\mu}\right)\right]}{\left[\log T_{h}^{-1}\left(\exp r^{\mu}\right)\right]^{\beta}}=B$,

a real number $>0$

for any $\alpha, \beta$ satisfying $\alpha>1,0<\beta<1, \alpha \beta>1$ and $0<\mu<$ $\rho_{g} \leq \infty$. Then

$$
\rho_{h}(f \circ g)=\infty .
$$

Proof. From (i) we have for all sufficiently large values of $r$ that

$$
\log T_{h}^{-1}\left(\exp \left(r^{\mu}\right)\right) \geq\left((A-\varepsilon) \log ^{[2]} r\right)^{\alpha}
$$

and from (ii) we obtain for all sufficiently large values of $r$ that

$$
\begin{aligned}
& \log \left[\frac{\log T_{h}^{-1}\left(T_{f}\left(\exp r^{\mu}\right)\right)}{\log T_{h}^{-1}\left(\exp r^{\mu}\right)}\right] \\
& \quad \geq(B-\varepsilon)\left[\log T_{h}^{-1}\left(\exp r^{\mu}\right)\right]^{\beta}
\end{aligned}
$$

that is,

$$
\begin{aligned}
& \frac{\log T_{h}^{-1}\left(T_{f}\left(\exp r^{\mu}\right)\right)}{\log T_{h}^{-1}\left(\exp r^{\mu}\right)} \\
& \quad \geq \exp \left[(B-\varepsilon)\left[\log T_{h}^{-1}\left(\exp r^{\mu}\right)\right]^{\beta}\right] .
\end{aligned}
$$

As $T_{h}^{-1}(r)$ is an increasing function of $r$, it follows from (26) and (27) and Lemma 1 for a sequence of values of $r$ tending to infinity that

$$
\frac{\log T_{h}^{-1} T_{f \circ g}(r)}{\log r} \geq \frac{\log T_{h}^{-1} T_{f}\left(\exp \left(r^{\mu}\right)\right)}{\log r}
$$

that is,

$$
\begin{aligned}
& \frac{\log T_{h}^{-1} T_{f \circ g}(r)}{\log r} \\
& \quad \frac{\log T_{h}^{-1} T_{f}\left(\exp \left(r^{\mu}\right)\right)}{\log T_{h}^{-1}\left(\exp \left(r^{\mu}\right)\right)} \cdot \frac{\log T_{h}^{-1}\left(\exp \left(r^{\mu}\right)\right)}{\log r}
\end{aligned}
$$

that is,

$$
\begin{aligned}
\frac{\log T_{h}^{-1} T_{f \circ g}(r)}{\log r} \\
\geq \exp \left[(B-\varepsilon)\left[\log T_{h}^{-1}\left(\exp r^{\mu}\right)\right]^{\beta}\right] \\
\quad \frac{(A-\varepsilon)\left(\log { }^{[2]} r\right)^{\alpha}}{\log r}
\end{aligned}
$$

that is,

$$
\begin{aligned}
\frac{\log T_{h}^{-1} T_{f \circ g}(r)}{\log r} \geq & \exp \left[(B-\varepsilon)(A-\varepsilon)^{\beta}\left(\log ^{[2]} r\right)^{\alpha \beta}\right] \\
& \cdot \frac{(A-\varepsilon)\left(\log ^{[2]} r\right)^{\alpha}}{\log r}
\end{aligned}
$$

that is,

$$
\begin{aligned}
& \frac{\log T_{h}^{-1} T_{f \circ g}(r)}{\log r} \\
& \geq \exp \left[(B-\varepsilon)(A-\varepsilon)^{\beta}\left(\log ^{[2]} r\right)^{\alpha \beta-1} \log ^{[2]} r\right] \\
& \quad \cdot \frac{(A-\varepsilon)\left(\log ^{[2]} r\right)^{\alpha}}{\log r}
\end{aligned}
$$

that is,

$$
\begin{array}{r}
\frac{\log T_{h}^{-1} T_{f \circ g}(r)}{\log r} \geq(\log r)^{(B-\varepsilon)(A-\varepsilon)^{\beta}\left(\log ^{[2]} r\right)^{\alpha \beta-1}} \\
\cdot \frac{(A-\varepsilon)\left(\log ^{[2]} r\right)^{\alpha}}{\log r}
\end{array}
$$

that is,

$$
\begin{aligned}
\limsup _{r \rightarrow \infty} & \frac{\log T_{h}^{-1} T_{f \circ g}(r)}{\log r} \\
\geq & \lim _{r \rightarrow \infty} \inf (\log r)^{(B-\varepsilon)(A-\varepsilon)^{\beta}\left(\log ^{[2]} r\right)^{\alpha \beta-1}} \\
& \cdot \frac{(A-\varepsilon)\left(\log ^{[2]} r\right)^{\alpha}}{\log r} .
\end{aligned}
$$

Since $\varepsilon(>0)$ is arbitrary and $\alpha>1, \alpha \beta>1$, the theorem follows from above. 
Theorem 4. Let $f$ be a meromorphic function and let $g, h$ be any two entire functions such that $0<\rho_{g}<\infty$ and $0<\lambda_{f}$. Further suppose that

(i) $\liminf _{r \rightarrow \infty} \frac{\log T_{h}^{-1}\left(\exp \left(r^{\mu}\right)\right)}{\left(\log ^{[2]} r\right)^{\alpha}}=A$, a real number $>0$,

(ii) $\liminf _{r \rightarrow \infty} \frac{\log \left[\log T_{h}^{-1}\left(T_{g}\left(\exp r^{\mu}\right)\right) / \log T_{h}^{-1}\left(\exp r^{\mu}\right)\right]}{\left[\log T_{h}^{-1}\left(\exp r^{\mu}\right)\right]^{\beta}}=B$, a real number $>0$

for any $\alpha, \beta$ satisfying $\alpha>1,0<\beta<1, \alpha \beta>1$, and $0<\mu<$ $\rho_{g}$. Then

$$
\rho_{h}(f \circ g)=\infty \text {. }
$$

We omit the proof of Theorem 4 as it can be carried out in the line of Theorem 3 and with the help of Lemma 2.

Theorem 5. Let $f$ be a meromorphic function and let $g, h$ be any two entire functions such that $0<\rho_{g} \leq \infty$ and $\lambda_{h}(f)>0$. Then

$$
\rho_{h}(f \circ g)=\infty
$$

Proof. Suppose $0<\mu<\rho_{g} \leq \infty$.

As $T_{h}^{-1}(r)$ is an increasing function of $r$, we get from Lemma 1 for a sequence of values of $r$ tending to infinity that

$$
\log T_{h}^{-1} T_{f \circ g}(r) \geq \log T_{h}^{-1} T_{f}\left(\exp \left(r^{\mu}\right)\right)
$$

that is,

$$
\log T_{h}^{-1} T_{f \circ g}(r) \geq\left(\lambda_{h}(f)-\varepsilon\right) r^{\mu}
$$

that is,

$$
\frac{\log T_{h}^{-1} T_{f \circ g}(r)}{\log r} \geq \frac{\left(\lambda_{h}(f)-\varepsilon\right) r^{\mu}}{\log r}
$$

that is,

$$
\limsup _{r \rightarrow \infty} \frac{\log T_{h}^{-1} T_{f \circ g}(r)}{\log r} \geq \liminf _{r \rightarrow \infty} \frac{\left(\lambda_{h}(f)-\varepsilon\right) r^{\mu}}{\log r}
$$

that is,

$$
\rho_{h}(f \circ g)=\infty
$$

Thus the theorem follows.

In the line of Theorem 5, one can easily prove the following theorem.

Theorem 6. Let $f$ be a meromorphic function and let $g, h$ be any two entire functions such that $0<\min \left\{\lambda_{f}, \rho_{g}\right\}$ and $\lambda_{h}(g)>0$. Then

$$
\rho_{h}(f \circ g)=\infty \text {. }
$$

Theorem 7. Let $f$ be a meromorphic function and let $g, h$ be any two entire functions such that $0<\rho_{g} \leq \infty$ and $\lambda_{h}(f)>0$. Then

$$
\limsup _{r \rightarrow \infty} \frac{\log T_{h}^{-1} T_{f \circ g}(r)}{\log T_{h}^{-1} T_{f}(r)}=\infty
$$

Proof. In view of Theorem 5, we obtain that

$$
\begin{array}{r}
\limsup _{r \rightarrow \infty} \frac{\log T_{h}^{-1} T_{f \circ g}(r)}{\log T_{h}^{-1} T_{f}(r)} \geq \limsup _{r \rightarrow \infty} \frac{\log T_{h}^{-1} T_{f \circ g}(r)}{\log r} \\
\cdot \lim _{r \rightarrow \infty} \inf \frac{\log r}{\log T_{h}^{-1} T_{f}(r)}
\end{array}
$$

that is,

$$
\limsup _{r \rightarrow \infty} \frac{\log T_{h}^{-1} T_{f \circ g}(r)}{\log T_{h}^{-1} T_{f}(r)} \geq \rho_{h}(f \circ g) \cdot \frac{1}{\rho_{h}(f)}
$$

that is,

$$
\limsup _{r \rightarrow \infty} \frac{\log T_{h}^{-1} T_{f \circ g}(r)}{\log T_{h}^{-1} T_{f}(r)}=\infty
$$

Thus the theorem follows.

Theorem 8. Let $f$ be a meromorphic function and let $g, h$ be any two entire functions such that $0<\min \left\{\lambda_{f}, \rho_{g}\right\}$ and $\lambda_{h}(g)>0$. Then

$$
\limsup _{r \rightarrow \infty} \frac{\log T_{h}^{-1} T_{f \circ g}(r)}{\log T_{h}^{-1} T_{g}(r)}=\infty .
$$

The proof of Theorem 8 is omitted as it can be carried out in the line of Theorem 7 and in view of Theorem 6.

Theorem 9. Let $f$ be a meromorphic function and let $h$ be an entire functions such that $0<\lambda_{h}(f) \leq \rho_{h}(f)<\infty$. Also let $g$ be an entire function with nonzero order. Then for every positive constant $A$ and every real number $\alpha$,

$$
\limsup _{r \rightarrow \infty} \frac{\log T_{h}^{-1} T_{f \circ g}(r)}{\left\{\log T_{h}^{-1} T_{f}\left(r^{A}\right)\right\}^{1+\alpha}}=\infty
$$

Proof. If $\alpha$ is such that $1+\alpha \leq 0$, then the theorem is trivial. So we suppose that $1+\alpha>0$.

Since $T_{h}^{-1}(r)$ is an increasing function of $r$, we get from Lemma 1 for a sequence of values of $r$ tending to infinity that

$$
\log T_{h}^{-1} T_{f \circ g}(r) \geq \log T_{h}^{-1} T_{f}\left(\exp \left(r^{\mu}\right)\right)
$$

that is,

$$
\log T_{h}^{-1} T_{f \circ g}(r) \geq\left(\lambda_{h}(f)-\varepsilon\right) r^{\mu},
$$

where we choose $0<\mu<\rho_{g} \leq \infty$. 
Again from the definition of $\rho_{h}(f)$, it follows for all sufficiently large values of $r$ that

$$
\log T_{h}^{-1} T_{f}\left(r^{A}\right) \leq\left(\rho_{h}(f)+\varepsilon\right) A \log r
$$

that is,

$$
\left\{\log T_{h}^{-1} T_{f}\left(r^{A}\right)\right\}^{1+\alpha} \leq\left(\rho_{h}(f)+\varepsilon\right)^{1+\alpha} A^{1+\alpha}(\log r)^{1+\alpha} .
$$

Now from (38) and (39), it follows for a sequence of values of $r$ tending to infinity that

$$
\frac{\log T_{h}^{-1} T_{f \circ g}(r)}{\left\{\log T_{h}^{-1} T_{f}\left(r^{A}\right)\right\}^{1+\alpha}} \geqslant \frac{\left(\lambda_{h}(f)-\varepsilon\right) r^{\mu}}{\left(\rho_{h}(f)+\varepsilon\right)^{1+\alpha} A^{1+\alpha}(\log r)^{1+\alpha}} .
$$

Since $r^{\mu} /(\log r)^{1+\alpha} \rightarrow \infty$ as $r \rightarrow \infty$, the theorem follows from above.

In the line of Theorem 9 and with the help of Lemma 2, one may state the following theorem without its proof.

Theorem 10. Let $f$ be a meromorphic function with nonzero finite lower order and let $g$ be an entire function with nonzero finite order. Also let $h$ be an entire function such that $\rho_{h}(f)<$ $\infty$ and $\lambda_{h}(g)>0$. Then for every positive constant $A$ and every real number $\alpha$,

$$
\limsup _{r \rightarrow \infty} \frac{\log T_{h}^{-1} T_{f \circ g}(r)}{\left\{\log T_{h}^{-1} T_{f}\left(r^{A}\right)\right\}^{1+\alpha}}=\infty .
$$

Theorem 11. Let $f$ be a meromorphic function and let $g$ be an entire function with nonzero order. Also let $h$ be an entire function such that $0<\lambda_{h}(f)$ and $\rho_{h}(g)<\infty$. Then for every positive constant $A$ and every real number $\alpha$,

$$
\limsup _{r \rightarrow \infty} \frac{\log T_{h}^{-1} T_{f \circ g}(r)}{\left\{\log T_{h}^{-1} T_{g}\left(r^{A}\right)\right\}^{1+\alpha}}=\infty .
$$

Theorem 12. Let $f$ be a meromorphic function with nonzero finite lower order and let $g$ be an entire function with nonzero finite order. Also let $h$ be an entire function such that $0<$ $\lambda_{h}(g) \leq \rho_{h}(g)<\infty$. Then for every positive constant $A$ and every real number $\alpha$,

$$
\limsup _{r \rightarrow \infty} \frac{\log T_{h}^{-1} T_{f \circ g}(r)}{\left\{\log T_{h}^{-1} T_{g}\left(r^{A}\right)\right\}^{1+\alpha}}=\infty .
$$

We omit the proof of Theorems 11 and 12 as those can be carried out in the line of Theorems 9 and 10, respectively.

Theorem 13. Let $f$ be a meromorphic function with nonzero finite order and lower order. Also let $g, h$ be any two entire functions such that $0<\lambda_{h}(f) \leq \rho_{h}(f)<\infty$. Then for every positive constant $\mu$ and each $\alpha \in(-\infty, \infty)$,

$$
\liminf _{r \rightarrow \infty} \frac{\left\{\log T_{h}^{-1} T_{f \circ g}(r)\right\}^{1+\alpha}}{\log T_{h}^{-1} T_{f}\left(\exp \left(r^{\mu}\right)\right)}=0 \quad \text { if } \mu>\lambda_{g} .
$$

Proof. If $1+\alpha \leq 0$, then the theorem is obvious. We consider $1+\alpha>0$.

Since $T_{h}^{-1}(r)$ is an increasing function of $r$, it follows from Lemma 3 for a sequence of values of $r$ tending to infinity that

$$
\log T_{h}^{-1} T_{f \circ g}(r)<\log T_{h}^{-1} T_{f}\left(\exp \left(r^{\mu}\right)\right)
$$

that is,

$$
\log T_{h}^{-1} T_{f \circ g}(r)<\left(\rho_{h}(f)+\varepsilon\right) r^{\mu} .
$$

Again for all sufficiently large values of $r$ we get that

$$
\log T_{h}^{-1} T_{f}\left(\exp \left(r^{\mu}\right)\right) \geq\left(\lambda_{h}(f)-\varepsilon\right) r^{\mu}
$$

Hence for a sequence of values of $r$ tending to infinity, we obtain from (45) and (46) that

$$
\frac{\left\{\log T_{h}^{-1} T_{f \circ g}(r)\right\}^{1+\alpha}}{\log T_{h}^{-1} T_{f}\left(\exp \left(r^{\mu}\right)\right)} \leq \frac{\left(\rho_{h}(f)+\varepsilon\right)^{1+\alpha} r^{\mu(1+\alpha)}}{\left(\lambda_{h}(f)-\varepsilon\right) r^{\mu}} .
$$

So from (47) we obtain that

$$
\liminf _{r \rightarrow \infty} \frac{\left\{\log T_{h}^{-1} T_{f \circ g}(r)\right\}^{1+\alpha}}{\log T_{h}^{-1} T_{f}\left(\exp \left(r^{\mu}\right)\right)}=0 .
$$

This proves the theorem.

Theorem 14. Let $f$ be a meromorphic function with nonzero finite order and lower order. Also let $g, h$ be any two entire functions such that $\rho_{h}(f)<\infty$ and $\lambda_{h}(g)>0$. Then for every positive constant $\mu$ and each $\alpha \in(-\infty, \infty)$,

$$
\liminf _{r \rightarrow \infty} \frac{\left\{\log T_{h}^{-1} T_{f \circ g}(r)\right\}^{1+\alpha}}{\log T_{h}^{-1} T_{g}\left(\exp \left(r^{\mu}\right)\right)}=0 \quad \text { if } \mu>\lambda_{g} .
$$

The proof is omitted as it can be carried out in the line of Theorem 13.

Theorem 15. Let $f$ be a meromorphic function with finite order and let $g$ be an entire function with nonzero finite lower order. Also let $h$ be another entire function such that $\lambda_{h}(f)>0$ and $\rho_{h}(g)<\infty$. Then for every positive constant $\mu$ and each $\alpha \in(-\infty, \infty)$,

$$
\liminf _{r \rightarrow \infty} \frac{\left\{\log T_{h}^{-1} T_{f \circ g}(r)\right\}^{1+\alpha}}{\log T_{h}^{-1} T_{f}\left(\exp \left(r^{\mu}\right)\right)}=0 \quad \text { if } \mu>\lambda_{g} .
$$

Theorem 16. Let $f$ be a meromorphic function with finite order and let $g$ be an entire function with nonzero finite lower order. Also let $h$ be another entire function such that $0<$ $\lambda_{h}(g) \leq \rho_{h}(g)<\infty$. Then for every positive constant $\mu$ and each $\alpha \in(-\infty, \infty)$,

$$
\liminf _{r \rightarrow \infty} \frac{\left\{\log T_{h}^{-1} T_{f \circ g}(r)\right\}^{1+\alpha}}{\log T_{h}^{-1} T_{g}\left(\exp \left(r^{\mu}\right)\right)}=0 \text { if } \mu>\lambda_{g} .
$$


We omit the proofs of Theorems 15 and 16 as those can be carried out in the line of Theorems 13 and 14, respectively, and with the help of Lemma 4.

Theorem 17. Let $f$ be a meromorphic function and let $g, h$ be any two entire functions such that $\rho_{h}(f)<\infty$ and $\lambda_{h}(f \circ g)=$ $\infty$. Then for every $\mu(>0)$,

$$
\lim _{r \rightarrow \infty} \frac{\log T_{h}^{-1} T_{f \circ g}(r)}{\log T_{h}^{-1} T_{f}\left(r^{\mu}\right)}=\infty .
$$

Proof. If possible, let there exist a constant $\beta$ such that for a sequence of values of $r$ tending to infinity,

$$
\log T_{h}^{-1} T_{f \circ g}(r) \leq \beta \cdot \log T_{h}^{-1} T_{f}\left(r^{\mu}\right) .
$$

Again from the definition of $\rho_{h}(f)$, it follows for all sufficiently large values of $r$ that

$$
\log T_{h}^{-1} T_{f}\left(r^{\mu}\right) \leq\left(\rho_{h}(f)+\varepsilon\right) \mu \log r .
$$

Now combining (53) and (54), we have for a sequence of values of $r$ tending to infinity that

$$
\log T_{h}^{-1} T_{f \circ g}(r) \leq \beta \cdot\left(\rho_{h}(f)+\varepsilon\right) \mu \cdot \log r
$$

that is,

$$
\lambda_{h}(f \circ g) \leq \beta \cdot \mu\left(\rho_{h}(f)+\varepsilon\right),
$$

which contradicts the condition $\lambda_{h}(f \circ g)=\infty$.

So for all sufficiently large values of $r$ we get that

$$
\log T_{h}^{-1} T_{f \circ g}(r) \geq \beta \cdot \log T_{h}^{-1} T_{f}\left(r^{\mu}\right),
$$

from which the theorem follows.

Remark 18. Theorem 16 is also valid with "limit superior" instead of "limit" if $\lambda_{h}(f \circ g)=\infty$ is replaced by $\rho_{h}(f \circ g)=\infty$ and the other conditions remain the same.

Corollary 19. Under the assumptions of Theorem 16 and Remark 18,

$$
\lim _{r \rightarrow \infty} \frac{T_{h}^{-1} T_{f \circ g}(r)}{T_{h}^{-1} T_{f}\left(r^{\mu}\right)}=\infty, \quad \limsup _{r \rightarrow \infty} \frac{T_{h}^{-1} T_{f \circ g}(r)}{T_{h}^{-1} T_{f}\left(r^{\mu}\right)}=\infty,
$$

respectively hold.

Proof. By Theorem 16, we obtain for all sufficiently large values of $r$ and for $K>1$ that

$$
\log T_{h}^{-1} T_{f \circ g}(r) \geq K \log T_{h}^{-1} T_{f}\left(r^{\mu}\right)
$$

that is,

$$
T_{h}^{-1} T_{f \circ g}(r) \geq\left\{T_{h}^{-1} T_{f}\left(r^{\mu}\right)\right\}^{K},
$$

from which the first part of the corollary follows.

Similarly using Remark 18, we obtain the second part of the corollary.
Analogously one may state the following theorem and corollaries without their proofs as those can be carried out in the line of Remark 18, Theorem 16, and Corollary 19, respectively.

Theorem 20. If $f$ is a meromorphic function and $g, h$ are any two entire functions such that $\rho_{h}(g)<\infty$ and $\rho_{h}(f \circ g)=\infty$, then for every $\mu(>0)$,

$$
\limsup _{r \rightarrow \infty} \frac{\log T_{h}^{-1} T_{f \circ g}(r)}{\log T_{h}^{-1} T_{g}\left(r^{\mu}\right)}=\infty .
$$

Corollary 21. Theorem 17 is also valid with "limit" instead of "limit superior" if $\rho_{h}(f \circ g)=\infty$ is replaced by $\lambda_{h}(f \circ g)=\infty$ and the other conditions remain the same.

Corollary 22. Under the assumptions of Theorem 17 and Corollary 21,

$$
\limsup _{r \rightarrow \infty} \frac{T_{h}^{-1} T_{f \circ g}(r)}{T_{h}^{-1} T_{g}\left(r^{\mu}\right)}=\infty, \quad \lim _{r \rightarrow \infty} \frac{T_{h}^{-1} T_{f \circ g}(r)}{T_{h}^{-1} T_{g}\left(r^{\mu}\right)}=\infty,
$$

respectively hold.

\section{Conclusion}

The notion of order and lower order which are the main tools to study the comparative growth properties of entire and meromorphic functions is very much classical in complex analysis. On the basis of the order and lower order of entire or meromorphic functions, several researchers have already explored their works on the area of comparative growth rates of composite entire and meromorphic functions in different directions. In fact, the main aim of this paper is actually to generalize the growth estimates of composite entire and meromorphic functions on the basis of their relative orders and relative lower orders with respect to an entire function. Moreover, the treatment of these notions may also be done by taking the help of slowly changing functions in order to study some different growth properties of composite entire and meromorphic functions.

\section{Conflict of Interests}

The authors declare that there is no conflict of interests regarding the publication of this paper.

\section{Acknowledgment}

The authors are thankful to the referee for his/her valuable suggestions towards the improvement of the paper.

\section{References}

[1] W. K. Hayman, Meromorphic Functions, The Clarendon Press, Oxford, UK, 1964.

[2] G. Valiron, Lectures on the General Theory of Integral Functions, Chelsea Publishing, New York, NY, USA, 1949. 
[3] L. Bernal, Crecimiento relativo de funciones enteras. Contribución al estudio de lasfunciones enteras con índice exponencial finito [Doctoral Dissertation], University of Seville, Sevilla, Spain, 1984.

[4] L. Bernal, "Orden relativo de crecimiento de funciones enteras," Collectanea Mathematica, vol. 39, no. 3, pp. 209-229, 1988.

[5] E. C. Titchmarsh, The Theory of Functions, Oxford University Press, Oxford, UK, 2nd edition, 1968.

[6] B. K. Lahiri and D. Banerjee, "Relative order of entire and meromorphic functions," Proceedings of the National Academy of Sciences, India A, vol. 69, no. 3, pp. 339-354, 1999.

[7] W. Bergweiler, "On the growth rate of composite meromorphic functions," Complex Variables, Theory and Application, vol. 14, no. 1-4, pp. 187-196, 1990.

[8] I. Lahiri and D. K. Sharma, "Growth of composite entire and meromorphic functions," Indian Journal of Pure and Applied Mathematics, vol. 26, no. 5, pp. 451-458, 1995.

[9] S. K. Datta and T. Biswas, "On a result of Bergweiler," International Journal of Pure and Applied Mathematics, vol. 51, no. 1, pp. 33-37, 2009. 


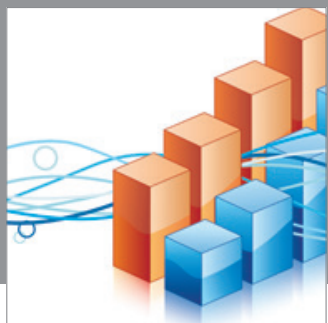

Advances in

Operations Research

mansans

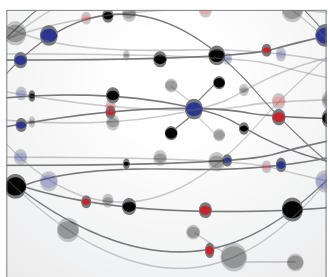

The Scientific World Journal

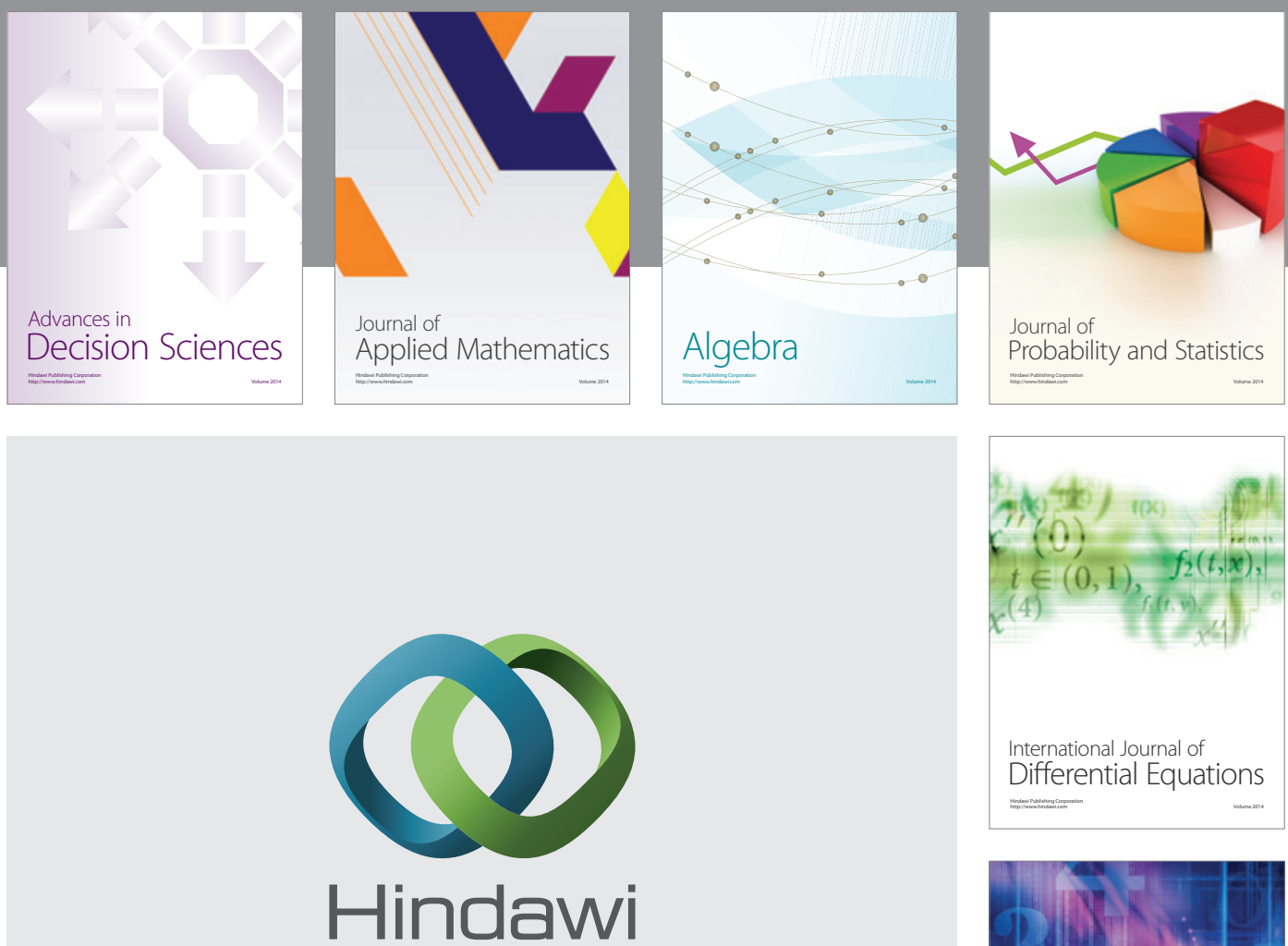

Submit your manuscripts at http://www.hindawi.com
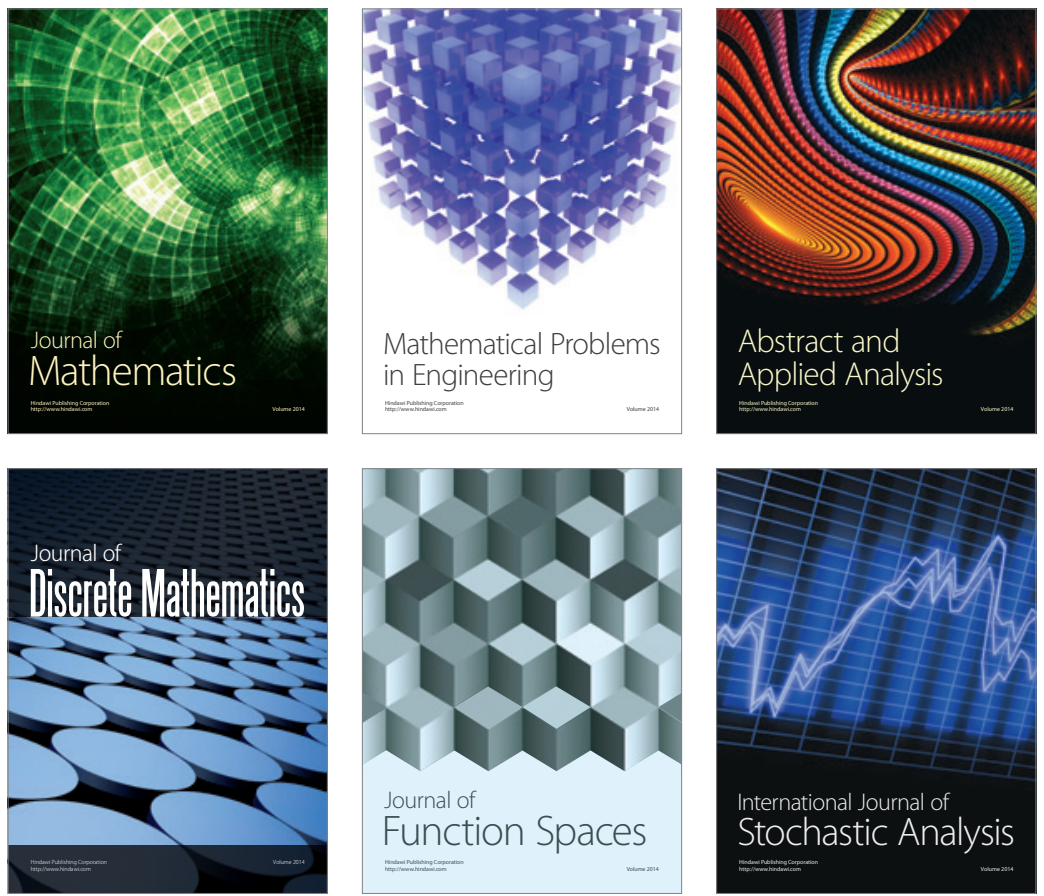

Journal of

Function Spaces

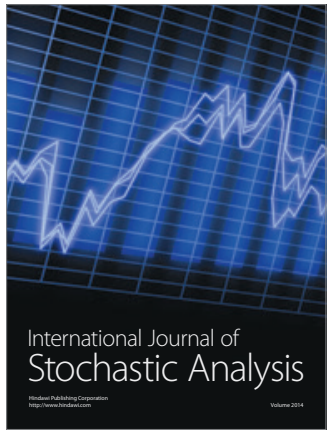

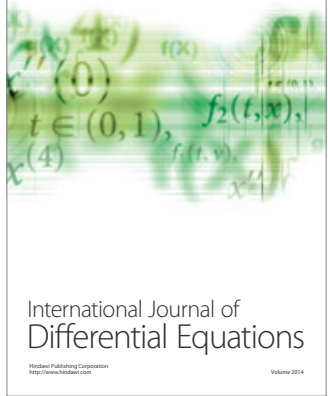
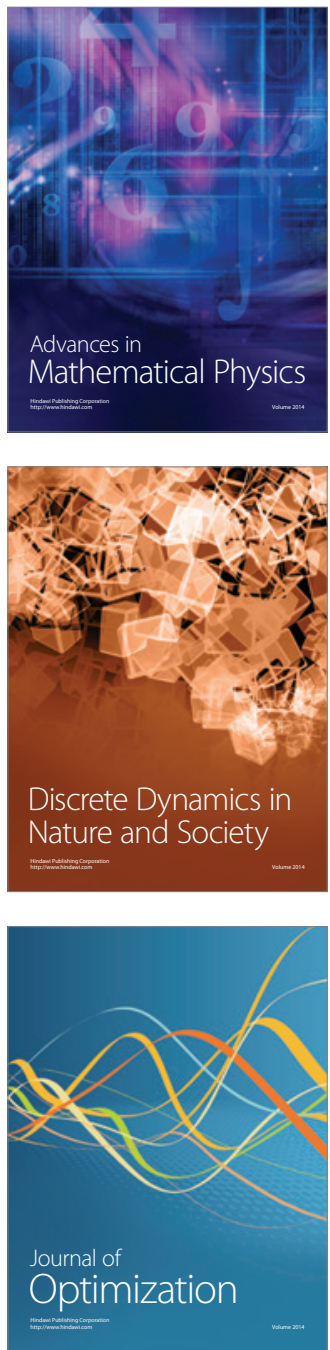